INSTITUT NATIONAL DE LA STATISTIQUE ET DES ETUDES ECONOMIQUES

Série des Documents de Travail du CREST

(Centre de Recherche en Economie et Statistique)

$n^{\circ}$ 2006-07

Un exercice de TVA sociale

S. GAUTHIER

Les documents de travail ne reflètent pas la position de l'INSEE et n'engagent que leurs auteurs.

Working papers do not reflect the position of INSEE but only the views of the authors.

${ }^{1}$ CREM, Université de Caen et CREST-LMA. Mail : gauthier@ensae.fr 


\title{
Un exercice de TVA sociale *
}

\author{
Stéphane Gauthier ${ }^{\dagger}$ \\ CREM, Université de Caen, et CREST
}

20 avril 2006

\begin{abstract}
Résumé
Ce texte étudie les effets d'une réforme de TVA sociale en équilibre général. Cette réforme consiste à substituer la TVA, une taxe assise principalement sur la consommation finale des ménages, à des cotisations sociales, assises sur les salaires et pour une part acquittées par les employeurs. Il propose une maquette analytique de l'économie française et la calibre à l'aide des données de 2003 issues de la comptabilité nationale. La maquette prédit qu'une baisse des cotisations sociales équivalente à 1 point de PIB environ, financée par la TVA, conduirait à une hausse de l'emploi se situant entre 400000 et 500000 individus sur un horizon de 10 ans si elle était concentrée sur la main-d'oeuvre peu qualifiée; elle aurait des effets beaucoup plus faibles, de 50000 à 100000 individus, si l'allègement de charges était étendu uniformément à la main-d'oeuvre qualifiée.
\end{abstract}

\section{Résumé en anglais}

This paper studies the consequences on employment of a fiscal reform called social VAT which consists to replace a wage tax by VAT. A calibration exercise based on 2003 french national accounts suggests that a 15 billions euros reduction of wage taxes, when financed by VAT, could lead to an increase in employment by 500000 individuals approximately, if it is concentrated on low skilled workers. However, if the wage tax rate is reduced in the same proportion for every type of labor, the rise in employment will strongly dampen; in this case, employment would increase by 100000 individuals at most.

Classification JEL : E62, H2.

Mots-clés : cotisations sociales, TVA, réforme fiscale.

\footnotetext{
* Je remercie Guy Laroque et Frédéric Tallet pour leurs très nombreux commentaires et suggestions. Je reste bien sûr le seul responsable des erreurs qui pourraient persister dans ce texte.

${ }^{\dagger}$ CREST-LMA Timbre J360, 15 bg Gabriel Péri, 92245 Malakoff cedex; Tel : 0141173738 ; Fax : 0141177666 ; Mail : gauthier@ensae.fr.
} 


\section{Introduction}

La protection sociale est financée aujourd'hui en France pour une grande part à l'aide des cotisations sociales : alors que 465 milliards d'euros de prestations de protection sociale ont été versés en 2003, les cotisations s'élevaient à 323 milliards d'euros ; le complément de financement est assuré, pour l'essentiel, par les impôts et taxes affectés à la protection sociale, à hauteur de 91.5 milliards d'euros, la CSG rapportant à elle seule environ 70 milliards d'euros.

Bien que l'impôt occupe une place encore minoritaire, son importance s'est accrue depuis une quinzaine d'années, puisqu'il représentait moins de $5 \%$ des recettes de la protection sociale en 1990. Cette tendance à la fiscalisation de la protection sociale a été parfois interprétée comme permettant de répondre à un désir social de faire coïncider plus étroitement les contributeurs et les bénéficiaires de ce système, mais elle est conçue plus souvent comme une composante importante de la politique de l'emploi en France. En partant du postulat que le coût du travail est trop élevé en France, différentes réformes ont en effet conduit, au cours des années 90, à une forte baisse des cotisations sociales à la charge des employeurs, notamment au niveau du SMIC.

L'efficacité de ces politiques a été longuement discutée ces dernières années ; on peut se référer à De Vreyer (2002) pour une synthèse. Mais, à l'exception du rapport d'Edmond Malinvaud pour le Conseil d'Analyse Economique en 1998, le mode de financement utilisé est en général passé sous silence ${ }^{1}$. L'objectif de ce texte est de décrire les effets d'une politique particulière, la TVA sociale, qui consiste à remplacer des cotisations sociales, assises sur les salaires et pour une part acquittées par les entreprises, par de la TVA, principalement assise sur la consommation des ménages.

Au regard de la théorie de l'incidence fiscale, l'efficacité de ce type de mesure est loin d'être acquise, une taxe sur le travail pouvant se révéler équivalente à une taxe sur la consommation (McLure, 1975). Toutefois, en pratique, ce résultat ne s'applique pas exactement, du fait de la définition de l'assiette effective de la TVA.

Pour quantifier les effets de la TVA sociale sur l'emploi, nous construisons une maquette analytique d'équilibre général faisant interagir le marché du travail et le marché des biens ${ }^{2}$. Au plan théorique, il est assez simple de retracer, dans les grandes lignes, la réaction de l'économie à une telle mesure. D'abord, bien sûr, la baisse des cotisations sociales conduit à une baisse du coût du travail à laquelle s'oppose la hausse de la TVA. Or, on le verra, l'une des contreparties du résultat

\footnotetext{
${ }^{1}$ Laffargue (2000) s'est également intéressé aux effets du financement d'un allègement de charges, notamment par une cotisation sur la valeur ajoutée, dans le cadre d'un modèle d'équilibre général calculable dont les caractéristiques essentielles sont, à l'exception de son caractère dynamique, assez similaires au cadre utilisé dans de ce texte.

${ }^{2}$ En ce sens, elle est assez différente de la maquette analytique de Salanié (2000), L'Horty (2000) ou Audric, Givord et Prost (2000), qui s'appuient uniquement sur la sphère de la production. Elle est plus proche du modèle d'équilibre général calculable de Laffargue (2000).
} 
d'équivalence fiscale est d'impliquer ici que le coût du travail, à l'équilibre, ne doit finalement pas changer. Si l'assiette de la TVA est plus large que celles des cotisations, la hausse du taux de TVA sera, au premier ordre, plus faible que la baisse du taux de cotisations, de sorte que le salaire réel doit augmenter pour maintenir le coût du travail constant. C'est cette hausse du salaire réel qui, en poussant la demande agrégée, amorce un processus multiplicateur au terme duquel la production et l'emploi seront plus élevés qu'initialement.

La calibration de la maquette suggère qu'en économie fermée, un allègement de 15 milliards d'euros, soit 1 point de PIB environ, conduirait à un horizon de 10 ans, lorsqu'il bénéficie à la main-d'oeuvre peu qualifiée, à une hausse de l'emploi légèrement supérieure à 400000 individus. Ces effets sont très nettement amoindris lorsque la plage de salaires concernée par l'allègement devient plus large : si les taux de cotisations sociales baissent tous dans la même proportion, l'emploi ne s'élève plus que de 50000 individus environ. Dans les deux cas, le coût du financement, lié à la hausse de la TVA, serait de l'ordre de 80000 individus. En économie ouverte, les effets sur l'emploi sont légèrement plus élevés : l'emploi augmenterait de 520000 individus lorsque l'allègement bénéficie seulement à la main-d'oeuvre non-qualifiée, et d'un peu plus de 100000 individus lorsque l'allègement est étendu à la main-d'oeuvre qualifiée.

La maquette est ensuite appliquée à une variante de la TVA sociale dans laquelle l'allègement de charges est cette fois-ci financé par une taxe pesant la valeur ajoutée, et non plus par la TVA; ce que l'on appelle parfois une cotisation sur la valeur ajoutée pour la distinguer de la TVA. C'est cette variante qui a été privilégiée dans les récentes annonces présidentielles sur ce sujet. Les mécanismes économiques en jeu sont alors de nature très différente, puisque l'imposition de la valeur ajoutée revient à taxer implicitement non seulement le travail, mais aussi le capital. La baisse induite du coût relatif du travail s'accompagne dans ce cas d'un effet de substitution favorable à l'emploi qui n'apparaît pas avec la TVA sociale, ou qui se limite à une substitution entre les différents types de main-d'oeuvre. Cependant, d'un autre côté, le découragement de la demande de capital tend à affaiblir la demande agrégée. Cet effet-volume, négatif, vient contrecarrer à l'effet de substitution, qui est lui positif : finalement, dans notre maquette au moins, la hausse de l'emploi est en fait sensiblement équivalente à celle que l'on obtiendrait avec la TVA sociale.

Ce texte est organisé de la façon suivante : dans une première partie (section 2), nous présentons un résultat de neutralité de la TVA sociale et nous isolons certaines circonstances qui, en pratique, devraient le remettre en cause; dans une seconde partie, nous élaborons une maquette analytique de l'économie calibrée pour décrire les effets de la suppression de environ 1 point de PIB de cotisations sociales (section 3 ). 


\section{TVA sociale et emploi}

Cette section s'appuie sur un résultat de neutralité d'une réforme substituant une taxe sur la consommation à une taxe assise sur la masse salariale pour examiner les canaux au travers desquels une telle politique est susceptible d'influencer l'emploi.

\subsection{Un résultat d'équivalence fiscale}

Considérons un modèle très simple dans lequel il y a trois agents (un ménage, une entreprise et l'Etat) et deux biens (un bien de consommation et le travail). L'entreprise produit le bien de consommation à partir du travail selon une technologie à rendements d'échelle constants : $y=\alpha l$ biens de consommation sont produits à l'aide de $l$ unités de travail. Sous cette hypothèse, les profits sont nuls à l'optimum : $p y-(1+t)(1+\tau) w l=0$, où $p$ est le prix-consommateur (le prix taxe comprise), $w$ le salaire net des cotisations sociales, $t$ le taux de taxe sur la consommation (le prix hors-taxe est donc $p /(1+t)$ ), et $\tau$ est le taux de cotisations sociales. De leur côté, les ménages consomment $c$ biens et offrent $l$ unités de travail. Leur contrainte budgétaire s'écrit $p c=w l+p T$, où $T$ est un transfert public. Soient $c=c^{d}(w / p, T)$ et $l=l^{s}(w / p, T)$ la demande de biens et l'offre de travail des ménages. Pour finir, l'Etat verse $T$ aux ménages et collecte des taxes. Son budget est supposé équilibré : $p T=t p y /(1+t)+\tau w l$.

Un équilibre de cette économie est un vecteur $(y, l, c, w, p, t)$ associé à $(\tau, T)$ tel que :

$$
\begin{gathered}
y=\alpha l, \\
p y=(1+t)(1+\tau) w l, \\
c=c^{d}(w / p, T), \\
l=l^{s}(w / p, T), \\
p c=w l+p T, \\
p T=t p y /(1+t)+\tau w l, \\
y=c .
\end{gathered}
$$

Il y a 6 inconnues et 7 équations. Mais, dans ce système, (3) et (5) impliquent (4), par l'identité de Walras; en outre, (2), (6) et (7) impliquent (5), par la loi de Walras. Enfin, étant données les propriétés d'homogénéité du système, seul le salaire réel $w / p$ est défini à l'équilibre. Aussi, sans perte de généralité, le bien de consommation sera choisi comme numéraire $(p=1)$. Avec cette convention, un équilibre est un vecteur $(y, l, c, w, t)$ associé à $(\tau, T)$ tel que :

$$
\begin{gathered}
y=\alpha l, \\
y=(1+t)(1+\tau) w l,
\end{gathered}
$$




$$
\begin{gathered}
y=c^{d}(w, T), \\
T=t y /(1+t)+\tau w l,
\end{gathered}
$$

Supposons que $t=\tau=0$ et que l'Etat s'engage dans une politique de TVA sociale. Cette politique est définie par une paire $(d t, d \tau)$ qui laisse le transfert réel $T$ inchangé. Elle consiste donc à modifier le taux de cotisations sociales d'un nombre donné de points et à ajuster le taux de TVA de sorte à maintenir inchangé le transfert réel à destination des ménages.

Ses conséquences s'obtiennent en différentiant le système formé par les équations (8) à (11) au point $t=\tau=0$ :

$$
\begin{gathered}
\hat{y}=\hat{l}, \\
\hat{y}=d t+d \tau+\hat{w}+\hat{l}, \\
\hat{y}=\varepsilon_{w}^{c} \hat{w}, \\
d t=-(w l / y) d \tau,
\end{gathered}
$$

où $\hat{y}=d y / y, \hat{l}=d l / l$, etc. La variable $\varepsilon_{w}^{c}$ représente l'élasticité de la demande de bien au salaire réel. Bien que son signe soit a priori ambigu, il est naturel de supposer que l'effet de substitution est dominant : une hausse du salaire réel conduit alors à une hausse de la consommation $\left(\varepsilon_{w}^{c}>0\right)$.

La résolution de ce système est simple. En utilisant (12) et (14), on obtient $\hat{y}=\hat{l}=\varepsilon_{w}^{c} \hat{w}$. Le taux de croissance du salaire réel est quant à lui donné par (12) et (13) : $\hat{w}=-(d t+d \tau)$. L'effet de la réforme sur l'emploi est donc positif si et seulement si $d t<-d \tau$, c'est-à-dire si le taux de TVA augmente moins que le taux de cotisations sociales ne baisse, ce qui est équivalent à $y>w l$, par (15). Mais cette condition n'est pas satisfaite : (9) implique en effet que la production $y$ est égale à la masse salariale $w l$ à l'équilibre initial. Il s'ensuit que $\hat{y}=\hat{l}=\hat{w}=0$. La TVA sociale reste ainsi sans effet sur les variables réelles à l'équilibre.

Ce résultat n'est pas surprenant. D'une part, une taxe sur la production est équivalente à la même taxe sur la masse salariale : le comportement de l'entreprise est identique dans les deux cas et l'Etat collecte la même la recette fiscale. D'autre part, nous avons réduit la TVA à une taxe sur la consommation. Or, ici, la consommation coïncide avec la production à l'équilibre, ce qui montre l'équivalence entre une taxe sur la consommation et une taxe sur la masse salariale.

\subsection{L'assiette de la TVA}

En pratique, bien sûr, la masse salariale ne se confond pas avec la production; dans le long terme, essentiellement parce que le travail n'est pas le seul facteur de production, de sorte que l'assiette des cotisations sociales est en fait 
plus étroite que la production nationale. De même, l'assiette de la TVA ne se confond pas non plus avec la production nationale. Elle se compose principalement des dépenses de consommation finale des ménages, de la formation brute de capital fixe des ménages et des administrations publiques, et des consommations intermédiaires des administrations publiques. En 2003, elle s'élevait à 980 milliards d'euros environ, soit $2 / 3$ du PIB seulement. En particulier, la TVA ne concerne pas la consommation finale des administrations publiques (380 milliards d'euros) et la formation brute de capital fixe des sociétés, financières ou non-financières (160 milliards d'euros); la TVA (nationale) taxe les importations, mais pas les exportations.

Pour décrire les conséquences de la divergence entre chacune de ces deux assiettes et le PIB, nous devons introduire dans le cadre de la section 2.1 le capital comme second facteur de production. Dans ce qui suit, on considèrera une fonction de production Cobb-Douglas à rendements d'échelle constants :

$$
y=k^{1-\alpha} l^{\alpha}
$$

avec $\alpha \in[0,1]$. Soient $r$ le taux d'intérêt $r$ et $\delta$ le taux de dépréciation du capital. Sous l'hypothèse de concurrence pure et parfaite, les conditions du premier ordre du problème de l'entreprise s'écrivent (cf. Annexe 1) :

$$
\begin{gathered}
\alpha y=(1+t)(1+\tau) w l, \\
(1-\alpha) y=(r+\delta) k .
\end{gathered}
$$

Ces deux conditions montrent qu'une hausse du taux de TVA est reportée sur le travail, dont le coût tend à s'élever, à salaire net et taux de cotisations donnés. Cette propriété résulte directement de la règle de non-imposition de l'investissement de l'entreprise à la TVA ; elle conduit à amoindrir la baisse du coût relatif du travail par rapport à celui du capital, et ainsi à limiter les possibilités de substitution du travail au capital.

Les revenus des ménages sont augmentés des revenus du patrimoine $r k$. Leur contrainte budgétaire s'écrit $c=w l+r k+T$. Soient

$$
c=c^{d}(w, r k+T)
$$

la fonction de consommation et $l=l^{s}(w, r k+T)$ l'offre de travail.

Enfin, la condition d'équilibre budgétaire s'écrit maintenant :

$$
T=t(y-i) /(1+t)+\tau w l,
$$

où $(y-i) /(1+t)$ représente la base de la TVA, et $t(y-i) /(1+t)$ la recette de la TVA.

Un équilibre de cette économie est un vecteur $(y, k, l, i, w, t)$ associé à $(\tau, T)$ tel que (16) à (20), et la condition d'équilibre sur le marché des biens

$$
y=c+i
$$


sont toutes satisfaites. Il y a 6 inconnues, et 5 équations seulement; il manque une équation pour que l'équilibre soit défini. On adoptera ici un point de vue de long terme en supposant simplement que

$$
i=\delta k
$$

c'est-à-dire que l'investissement n'est utilisé que pour maintenir le stock de capital constant.

Pour un taux d'intérêt $r$ donné, et en négligeant l'effet des variations du revenu non-salarial sur la consommation, les conséquences de la TVA sociale sont décrites par le système suivant :

$$
\begin{gathered}
\hat{y}=(1-\alpha) \hat{k}+\alpha \hat{l}, \\
\hat{y}=d t+d \tau+\hat{w}+\hat{l}, \\
\hat{y}=\hat{k}, \\
\hat{c}=\varepsilon_{w}^{c} \hat{w} \\
(y-i) d t=-w l d \tau \\
\hat{y}=(c / y) \hat{c}+(i / y) \hat{k}
\end{gathered}
$$

Pour résoudre ce système, remarquons tout d'abord que le taux d'intérêt et le taux de dépréciation du capital étant donnés, le coût marginal du capital, et ainsi la productivité marginale du capital qui lui est égale à l'optimum, ne varient pas avec le taux de TVA. Donc, le taux de croissance de la production, en réponse à la politique, doit être le même que celui du capital ; c'est ce qu'indique la condition (25). L'hypothèse de rendements constants implique alors immédiatement, par (23), que le taux de croissance de l'emploi doit être le même que celui du capital. Ainsi, la productivité marginale du travail ne varie pas, et le coût marginal du travail $(1+\tau) w l$ ne doit pas varier non plus; on a effectivement $\hat{w}=-(d t+d \tau)$ par (24). A ce stade, la politique reste donc neutre, comme elle l'était dans la section 2.1 .

L'efficacité de la politique va dépendre de l'effet du salaire réel, qui mesure ici le pouvoir d'achat du revenu du travail pour le ménage, sur la demande de biens. En fait, la condition (27) d'équilibre budgétaire de l'Etat montre que les ajustements de taux sont, au premier ordre, inversement liés au rapport des assiettes des deux prélèvements : $d t=-w l /(y-i) d \tau$. Il s'ensuit que le salaire réel augmente, lorsque le taux de cotisations sociales diminue, si et seulement si le taux de TVA augmente moins que le taux de cotisations ne baisse, ce qui est équivalent à $w l<y-i$; ou bien encore, les taux d'impositions étant nuls initialement, au fait que la base de la TVA, la consommation des ménages est plus large que celle des cotisations sociales.

Sous cette condition, la consommation augmente en proportion de l'élasticitéprix de la demande au salaire réel et du rapport des deux bases; par (26), on 
$\mathrm{a}: \hat{c}=-\varepsilon_{w}^{c}(1-w l /(y-i)) d \tau$. Cette hausse de la demande s'accompagne d'une hausse de la production, qui déclenche un processus multiplicateur, le taux de croissance de la production étant égal à celui de la demande de capital. Par (28), l'effet multiplicateur sera d'autant plus grand que $i / y$ est grand. On a finalement :

$$
\begin{gathered}
(1-i / y) \hat{l}=-(c / y) \varepsilon_{w}^{c}(1-w l /(y-i)) d \tau \\
\Leftrightarrow \hat{l}=-\varepsilon_{w}^{c}(1-w l / c) d \tau .
\end{gathered}
$$

Pour résumer, l'effet sur l'emploi résulte in fine d'un seul effet-volume : le salaire réel s'ajuste de sorte que le coût du travail reste inchangé. Il augmente lorsque l'assiette de la TVA est plus large que celle des cotisations, ce qui provoque une hausse de la consommation, de la production et de l'emploi; aucun effet de substitution capital-travail ne rentre en jeu, le travail augmentant dans la même proportion que le capital. Par conséquent, plus l'assiette des cotisations sociales est large par rapport à celle de la TVA, et plus l'élasticité de la demande au salaire réel élevée, plus l'effet de la politique sur l'emploi sera important.

\section{Une maquette analytique}

$\mathrm{Au}$ moins trois hypothèses sont restrictives dans le cadre de la section 2.2. D'abord, les taux d'imposition ne sont pas nuls lorsque la politique est mise en place, de sorte que les changements de taux d'imposition s'accompagnent de modifications des assiettes fiscales qui ne peuvent pas être négligées. Ensuite, certains ajustements sur les marchés se font par les quantités, et non par les prix; par exemple, du chômage subsiste sur le marché du travail, et ce chômage touche préférentiellement la main-d'oeuvre peu qualifiée sur laquelle les allègements de charges ont été concentrés durant les années 90. Enfin, l'élasticité de l'offre de travail au salaire est souvent considérée comme faible, voire nulle.

On distinguera dorénavant le travail qualifié du travail peu qualifié; tous les deux seront offerts en quantités fixes et, suivant Laffargue (2000) ou Salanié (2000), la main-d'oeuvre peu qualifiée sera touchée par du chômage de type classique, ce qui tend bien sûr à favoriser l'efficacité attendue d'une baisse du coût du travail.

\subsection{Les comportements}

On considère une fonction de production Cobb-Douglas $y=f\left(k, l_{q}, l_{n q}\right)$ à rendements constants, dans laquelle $l_{q}$ (resp., $l_{n q}$ ) représente l'emploi qualifié (resp., non-qualifié). On notera $\alpha_{q}$ (resp., $\alpha_{n q}$ ) l'élasticité de la production à l'emploi qualifié (resp., non-qualifié).

Les ménages qualifiés et non-qualifiés ne diffèrent que par leur ressources. Leurs préférences sont décrites par

$$
u(c, m)=B c^{1-1 / \xi} /(1-1 / \xi)+m, \quad B>0,
$$


où $m$ une demande de monnaie. Leur contrainte budgétaire s'écrit $p c+m \leq R$, où $R$ comprend l'ensemble des revenus des ménages. L'homogénéité des préférences des ménages permet d'écrire une fonction de consommation agrégée $c=\eta p^{-\xi}$, où $\eta=B^{\xi}>0$ et $\xi(\xi \geq 0)$ représente l'élasticité de la demande de bien au prix-consommateur.

L'Etat est supposé acheter une quantité exogène $\gamma$ de biens intermédiaires soumis à TVA. La recette de la TVA s'élève donc à $t p(c+\gamma) /(1+t)$; dans ce qui suit, $c+\gamma$ synthétisera en fait la base fiscale de la TVA, et $\gamma$ sera interprétée comme la base de la TVA nette de la consommation finale des ménages. La recette fiscale totale s'écrit ainsi

$$
t p(c+\gamma) /(1+t)+\tau_{q} w_{q} l_{q}+\tau_{n q} w_{n q} l_{n q},
$$

où $\tau_{q}$ et $\tau_{n q}$ sont les taux de cotisations sociales sur les salaires $w_{q} l_{q}$ des qualifiés et $w_{n q} l_{n q}$ des non-qualifiés, respectivement.

\subsection{L'équilibre}

L' entreprise n'est pas contrainte, ni sur le marché des biens de consommation, ni sur le marché des facteurs de production :

$$
\begin{gathered}
y=f\left(k, l_{q}, l_{n q}\right), \\
\left(1-\alpha_{q}-\alpha_{n q}\right) p y=(r+\delta) k, \\
\alpha_{q} p y=(1+t)\left(1+\tau_{q}\right) w_{q} l_{q}, \\
\alpha_{n q} p y=(1+t)\left(1+\tau_{n q}\right) \bar{w}_{n q} l_{n q} .
\end{gathered}
$$

Alors que les ménages qualifiés ne subissent pas non plus de contraintes, les ménages non-qualifiés sont quant à eux contraints sur le marché du travail, où subsiste un excès d'offre. La forme de la fonction d'utilité choisie implique toutefois que cette contrainte ne va peser que sur la demande de monnaie, puisque la consommation des ménages non-qualifiés est indépendante de leur revenu. L'équilibre sur le marché des biens s'écrit donc :

$$
y=c+i+g+\gamma
$$

où

$$
c=\eta p^{-\xi}
$$

et

$$
i=\delta k \text {. }
$$

L'offre de travail étant inélastique, on a enfin :

$$
l_{q}=\bar{l}_{q}
$$

Ainsi, pour résumer, un équilibre est un vecteur $\left(y, k, l_{q}, l_{n q}, w_{q}, p, c, i\right)$ associé à la politique fiscale $\left(t, \tau_{q}, \tau_{n q}\right)$ et satisfaisant les 8 équations (30) à (37). 
Lorsque l'Etat décide de modifier simultanément les taux de cotisations sociales pour des montants $d \tau_{q}$ et $d \tau_{n q}$, les cotisations sociales nominales varient en réponse de $d\left(\tau_{q} w_{q} l_{q}+\tau_{n q} w_{n q} l_{n q}\right)$. Cette variation est intégralement compensée par un ajustement adéquat $d t$ du taux (apparent) de TVA. Soit :

$$
d(t p(c+\gamma) /(1+t))+d\left(\tau_{q} w_{q} l_{q}+\tau_{n q} w_{n q} l_{n q}\right)=0 .
$$

Les effets de la TVA sociale s'obtiennent en différentiant le système (30)(38). Après quelques simplifications, pour un taux d'intérêt $r$ donné, et pour une dépense publique en biens finals et intermédiaires $g+\gamma$ donnée, on obtient :

$$
\begin{gathered}
\hat{y}=\left(1-\alpha_{q}-\alpha_{n q}\right) \hat{k}+\alpha_{n q} \hat{l}_{n q}, \\
\hat{p}+\hat{y}=\hat{k} \\
\hat{p}+\hat{y}=\hat{t}+\hat{\tau}_{q}+\hat{w}_{q}, \\
\hat{p}+\hat{y}=\hat{t}+\hat{\tau}_{n q}+\hat{l}_{n q} \\
\hat{y}=-\xi(c / y) \hat{p}+(i / y) \hat{k}
\end{gathered}
$$

auxquelles se rajoute la condition (38).

La résolution du système composé de (39) à (43) donne, en particulier,

$$
\left((y-i)-\alpha_{q}(y-\xi c)\right) \hat{l}_{n q}=-\left((y-i)-\left(\alpha_{q}+\alpha_{n q}\right)(y-\xi c)\right)\left(\hat{t}+\hat{\tau}_{n q}\right) .
$$

En pratique, le coût du travail qualifié est plus important que la formation brute de capital fixe des entreprises, de sorte que $(y-i)-\alpha_{q}(y-\xi c)>0$. Lorsque la part de la rémunération du travail non-qualifié dans la production est suffisamment faible, le membre de gauche de (44) est du signe opposé à celui de $\hat{t}+\hat{\tau}_{n q}$. En d'autres termes, l'emploi augmente si et seulement si la baisse du taux de cotisations sociales sur la main-d'oeuvre non-qualifiée est plus importante que la hausse du taux de TVA qui la finance, ou bien encore, au premier ordre, si l'assiette des cotisations sociales prélevées sur les non-qualifiés est plus étroite que celle de la TVA, une condition qu'il est d'autant plus vraisemblable de voir satisfaite que la réduction de charges est précisément ciblée.

Il est facile de vérifier qu'une hausse de l'investissement amoindrit l'efficacité de la mesure sur l'emploi si et seulement si $y>\xi c$. Intuitivement, une hausse de l'investissement a un effet positif associé à la demande agrégée, mais aussi un effet négatif, parce qu'elle réduit l'assiette de la TVA (pour une production donnée) ; l'effet négatif sera dominé si la consommation réagit peu à la hausse du taux de TVA, c'est-à-dire $\xi$ suffisamment petit. Au contraire, une hausse de la consommation amplifie systématiquement l'effet de la mesure sur l'emploi : non seulement la hausse de la demande est plus importante, mais la base de la TVA est plus large, ce qui contient la hausse du taux de TVA. Les conséquences d'une hausse de la production sont quant à elles incertaines; on peut vérifier que l'emploi s'élève, pour $\hat{t}+\hat{\tau}_{n q}<0$, si et seulement si $\alpha_{n q}(y-i)<\left(1-\alpha_{q}\right)(y-\xi c)$, ce qui est vrai pour $\xi$ suffisamment petit, et dès lors que la part de la rémunération du travail non-qualifié est plus faible que celle du capital. 


\subsection{Données de calibration}

On retient le champ des sociétés non-financières, des sociétés financières et des ménages. Toutes les données agrégées sont issues des comptes nationaux 2003. Pour ce qui concerne la distinction entre les différents types de main-d'oeuvre, on utilisera Skalitz (2004), bien qu'elle s'applique à un champ légèrement différent (les branches EA à ER de la Nomenclature Economique de Synthèse). Seront considérés comme non-qualifiés les ouvriers non-qualifiés et tous les employés. Sur cette base, il y $2 / 3$ d'individus qualifiés et $1 / 3$ d'individus non-qualifiés, et le salaire brut des qualifiés représente $73 \%$ du salaire brut total du champ.

L'emploi total s'élève à 18.5 millions d'individus. Il est ventilé au prorata de l'emploi qualifié et non-qualifié dans les branches EA à ER de la NES.

Soit : $l_{q}=12.2, l_{n q}=6.3$ (millions d'individus).

Les cotisations sociales des employeurs (effectives et imputées) s'élèvent à 153.5 milliards d'euros; les cotisations salariés à 61.5 milliards d'euros. Pour un coût du travail égal à 602.5 milliards d'euros, on en déduit la rémunération nette des cotisations sociales (employeurs et salariés confondus), 387.5 milliards d'euros.

Le salaire brut du champ s'élève à 449 milliards d'euros; en le ventilant à raison de $73 \%$ pour les qualifiés, on obtient un salaire brut des qualifiés égal à 327.8 milliards d'euros et à 121.2 milliards pour les non-qualifiés. Les cotisations sociales salariés s'élevant à 61.5 milliards d'euros, on obtient celles qui sont à la charge des salariés qualifiés en les ventilant au prorata des salaires bruts, soit 45 milliards pour les qualifiés et 16.5 pour les non-qualifiés. On en déduit les salaires nets des cotisations sociales : 283 milliards d'euros pour les qualifiés, et 104.5 pour les non-qualifiés.

Pour reconstituer les cotisations employeurs, on supposera que le taux $\tau_{q}$ est de $8 \%$ supérieur à $\tau_{n q}$ (Salanié (2000)). Alors, le taux de cotisations sociales pour les non-qualifiés est de $52 \%$, ce qui donne des cotisations sociales (employeurs et employés confondus) imputables aux non-qualifiés égales à 54.9 milliards d'euros. Et donc, par différence avec les salaires bruts, les cotisations employeurs sur les salaires non-qualifiés s'élèvent à 38.3 milliards d'euros.

Il s'ensuit que les cotisations sociales employeurs sur les salaires qualifiés sont égales à 115.2 milliards d'euros et le coût du travail qualifié se monte à 443 milliards d'euros. D'où le taux de $57 \%$ pour les cotisations des qualifiés.

$\mathrm{Au}$ total, $w_{q} l_{q}=283, w_{n q} l_{n q}=104.5$ (milliards d'euros), $\tau_{q}=0.57$ et $\tau_{n q}=0.52$.

La consommation finale des ménages, $p c$, s'élève à 868 milliards d'euros. L'assiette de la TVA et la recette de la TVA sont de 1088 milliards d'euros, de sorte que, par différence avec la consommation finale des ménages, $p \gamma$ est égal 
à 220 milliards d'euros. La TVA nette collectée étant de 110 milliards d'euros, le taux moyen apparent de TVA est de 10,1\%.

Il est difficile de donner une valeur précise à l'élasticité-prix $\xi$ de la consommation. Lhorty (2000) la pose égale à 0.5. Chauvin et alii (2004) l'estime à 0.8 pour les biens durables. Dans sa synthèse sur les politiques d'allègements de charges, de Vreyer (2002) donne une fourchette allant de 0 à 2. Malinvaud (1998) utilise même parfois une élasticité-prix de la demande agrégée à long terme égale à 4 . Nous suivrons ici Lhorty (2000), mais on donnera les résultats pour $\xi=1,2,4$.

On a donc : $p c=868, p \gamma=220, t=0.101$ et $\xi=0.5$.

Dans le champ retenu, le PIB est de 1151.5 milliards d'euros, et l'investissement, $i$, évalué au prix consommateur, est égal à 278 milliards d'euros. Sur la base d'une fonction de production agrégée Cobb-Douglas, les parts $\alpha_{q}$ et $\alpha_{n q}$ correspondent à des parts de rémunération (précisément, des rapports entre le coût du travail et le PIB évalué au prix hors taxe).

On a alors : $p y=1151.5, p i=278, \alpha_{q}=0.43$ et $\alpha_{n q}=0.15$.

Tableau récapitulatif

\begin{tabular}{|c|c|c|c|c|c|}
\hline$w_{q} l_{q}$ & $w_{n q} l_{n q}$ & & py & $p c$ & $p \gamma$ \\
\hline 283 & 104.5 & & 51.5 & 868 & 220 \\
\hline$t$ & $\tau_{q}$ & $\tau_{n q}$ & $\alpha_{q}$ & $\alpha_{n q}$ & $\xi$ \\
\hline 0.101 & 0.57 & 0.52 & 0.43 & 0.15 & 0.5 \\
\hline
\end{tabular}

\subsection{Eléments de chiffrage}

Pour obtenir quelques points de repères sur la maquette, on commence par évaluer les effets sur l'emploi de deux politiques non-financées, une expérience d'allègements de charges et un redressement du taux de TVA. Ensuite, les deux politiques sont mises en oeuvre simultanément au sein d'une réforme de TVA sociale.

Réduisons tout d'abord les cotisations sociales de 15 milliards d'euros ex ante, soit 1 point de PIB environ. Pour une baisse $\hat{\tau}_{n q}$ du taux de cotisations sociales sur les salaires des non-qualifiés, le taux de cotisations sur les salaires des qualifiés est supposé baisser de $\hat{\tau}_{q}=\beta \hat{\tau}_{n q}$ où $\beta$ est un paramètre libre. Lorsque $\beta=0$, la baisse est concentrée sur les non-qualifiés. Elle est uniforme pour $\beta=1$, et ne concerne que les qualifiés si $\beta$ est arbitrairement grand. Ex ante, la masse salariale est donnée, et l'on a donc :

$$
\left(\left(1+\tau_{n q}\right) w_{n q} l_{n q}+\beta\left(1+\tau_{q}\right) w_{q} l_{q}\right) \hat{\tau}_{n q}=-15 .
$$


Les effets de cette politique sur l'emploi sont décrits par (44) et (45), pour $\hat{t}=0$. Avec les données de la section 3.3, l'emploi non-qualifié s'élèverait de 480000 individus, lorsque la baisse des cotisations est concentrée sur la main-d'oeuvre non-qualifiée $(\beta=0)^{3}$. Il ne s'élèverait plus que de 130000 individus environ lorsque la baisse des cotisations est uniforme $(\beta=1)$.

Augmentons maintenant les recettes de la TVA de 15 milliards d'euros ex ante :

$$
p(c+\gamma) \hat{t} /(1+t)=15 \text {. }
$$

Les effets de cette réforme sont donnés par (44) et (46), pour $\hat{\tau}_{q}=\hat{\tau}_{n q}=0$. L'emploi non-qualifié baisserait alors de 80000 emplois environ.

On peut déduire les effets de la TVA sociale sur l'emploi de ce qui précède. Pour un allègement ex ante des cotisations sociales de 15 milliards d'euros financé par la TVA, (44), (45) et (46) doivent être satisfaites. Les résultats dépendent bien sûr de la plus ou moins grande concentration des allègements de charges sur l'emploi non-qualifié. Pour $\beta=0$, l'emploi non-qualifié augmenterait de 400000 individus environ. Pour $\beta=1$, il augmenterait approximativement de 50000 individus.

Remarque 1. Elasticité-prix de la demande. La littérature sur les allègements de charges met souvent l'accent sur deux paramètres critiques : l'élasticité de substitution entre le travail qualifié et le travail non-qualifié et l'élasticitéprix de la demande. Retenir une élasticité de substitution unitaire n'est pas déraisonnable, même si ce choix tend sans doute à surévaluer légèrement ${ }^{4}$ les capacités réelles de substitution d'un facteur de production à un autre en réponse aux modifications des prix relatifs des facteurs. Par contre, l'élasticité-prix de la demande, égale à $-1 / 2$, peut être jugée faible. Pour cette raison, le tableau ci-dessous donne la réaction de l'emploi non-qualifié $d l_{n q}$ pour différentes valeur du paramètre $\xi$.

$$
\begin{array}{cccc}
\xi & 1 & 2 & 4 \\
d l_{n q} & 470000 & 530000 & 590000
\end{array}
$$

Remarque 2. Economie ouverte. L'effet du solde de la balance commerciale $x-f$ n'a pas été pris en compte dans cette section. Les exportations $x$ dépendent

\footnotetext{
${ }^{3}$ Ces résultats sont plus élevés que ceux obtenus par Audric, Givord et Prost (2000) mais, contrairement à notre maquette, cette étude néglige l'effet de la hausse de l'emploi et des salaires sur la demande qui s'adresse aux entreprises; en outre, elle s'applique à un champ de 13.3 millions de salariés, qui est donc plus restreint que le notre. Ces résultats sont plus proches, mais restent supérieurs, aux résultats obtenus par Laffargue (2000) pour une élasticité de substitution plus élevée, 2.5, que celle utilisée dans notre maquette, où elle est fixée à 1.

${ }^{4}$ L'analyse théorique de la section 2 suggère en effet que la substitution du travail au capital ne joue pas un rôle important lorsque l'allègement de charges est financé par la TVA; seule la substitution entre la main-d'oeuvre qualifiée et la maind'oeuvre non-qualifiée rentrent en ligne de compte, et celle-ci est limitée, sous l'hypothèse que la quantité de travail qualifiée employée à l'équilibre est fixée.
} 
(positivement) du rapport $e(1+t) p^{*} / p$ tandis que les importations $f$ dépendent (négativement) de $e p^{*} / p$, où $e$ est le taux de changes et $p^{*}$ le prix-consommateur des biens étrangers. Dans un système de taux de changes fixes, et pour $p^{*}$ donné, on a donc $\hat{x}=-\phi_{x}(\hat{p}-\hat{t})$ et $\hat{f}=\phi_{f} \hat{p}$. L'équation d'équilibre sur le marché des biens devient alors :

$$
\hat{y}=-\xi(c / y) \hat{p}+(i / y) \hat{k}-(x / y) \phi_{x}(\hat{p}-\hat{t})+(f / y) \phi_{f} \hat{p}
$$

Elle doit être substituée à (43). Le système des équations d'équilibre reste sinon le même, et (44) se réécrit :

$$
\begin{aligned}
& \left((y-i)-\alpha_{q}\left(y-\xi c-x \phi_{x}+f \phi_{f}\right)\right) \hat{l}_{n q} \\
& \quad=-\left((y-i)-\left(\alpha_{q}+\alpha_{n q}\right)\left(y-\xi c-x \phi_{x}+f \phi_{f}\right)\right)\left(\hat{t}+\hat{\tau}_{n q}\right)+x \phi_{x} \hat{t}
\end{aligned}
$$

Etant donnée la définition de $\gamma$, les ajustements de taux ex ante sont encore donnés par (45) et (46).

Pour $x=407.7, f=390.2$ milliards d'euros, $\phi_{x}=1.1$ et $\phi_{f}=0.2$ (Hervé (2001)), dans le cas où $\xi=0.5$, une baisse de 15 milliards d'euros des cotisations sociales financée par une hausse de la TVA conduirait à une hausse de l'emploi de 520000 individus pour $\beta=0$, aurait un effet bien moindre pour $\beta=1$ (l'emploi augmenterait de 115000 individus). Elle conduirait à une baisse de l'emploi de 30000 pour $\beta$ suffisamment élevé. L'ouverture de l'économie améliorerait donc l'efficacité de la TVA sociale en termes d'emploi de 100000 individus au plus.

Remarque 3. Effet-revenu. L'évaluation néglige l'effet des variations de la production sur la consommation, l'investissement, et le solde de la balance commerciale. Il est possible qu'en renforçant l'effet-volume, la TVA sociale ait des effets plus important sur l'emploi que ceux qui ont été obtenus dans cette maquette.

Remarque 4. Taux apparent, taux normal et taux réduit. Les évaluations faites dans la section précédente impliquent le taux (moyen) apparent de TVA. Le cadre utilisé dans ce texte ne permet pas d'apprécier les effets de la TVA sociale si la base de substitution devait être restreinte aux seuls biens taxés au taux normal; de même, si la base de substitution cö̈ncidait avec les biens taxés au taux normal ou au taux réduit. Une réponse satisfaisante à cette question nécessiterait de distinguer plusieurs types de biens de consommation qui diffèrent selon le taux de TVA qui leur est appliqué.

\section{Cotisations sur la valeur ajoutée}

En reportant le financement des cotisations sociales sur l'assiette de la TVA, la réforme concerne en premier lieu le travail, le coût du capital n'étant pas affecté par la hausse de la TVA. Si l'assiette de substitution est plus large, qu'elle coïncide par exemple avec la valeur ajoutée, c'est-à-dire avec le PIB, la 
taxe est reportée à la fois sur le travail et sur le capital en réponse à la baisse des cotisations. Le problème de l'entreprise est alors décrit par (30), (32) et (33) ; la condition du premier ordre (31) devient

$$
\left(1-\alpha_{q}-\alpha_{n q}\right) p y=(1+t)(r+\delta) k
$$

ce qui montre que le coût marginal du capital augmente avec le taux $t$ de cotisations sur la valeur ajoutée. Les équations (34) à (37) sont inchangées, et la condition de maintien du transfert social agrégé à son niveau initial s'écrit désormais

$$
d\left(t y /(1+t)+\tau_{q} w_{q} l_{q}+\tau_{n q} w_{n q} l_{n q}\right)=0 .
$$

Cette condition vient remplacer (38).

Avec ce type de financement, le coût relatif du capital par rapport au travail augmente ; le coût du travail devrait légèrement baisser et celui du capital augmente, ce qui incite l'entreprise à substituer du travail à du capital. Les effets de la mesure sont encore décrits par le système d'équations (39) à (43), mais (40) est modifiée comme suit :

$$
\hat{p}+\hat{y}=\hat{t}+\hat{k}
$$

La résolution de ce système donne, en particulier,

$$
\left((y-i)-\alpha_{q}(y-\xi c)\right) \hat{l}_{n q}=-\xi c \hat{t}-\left((y-i)-\left(\alpha_{q}+\alpha_{n q}\right)(y-\xi c)\right) \hat{\tau}_{n q} .
$$

Dans cette formule, $\hat{t}$ et $\hat{\tau}_{n q}$ ne sont plus traités de façon symétrique parce que le taux $t$ s'applique à tous les facteurs de production alors que $\tau_{n q}$ ne concerne qu'une partie de la masse salariale. Avec les données de la section 3.3, et pour un taux $t$ de cotisations sur la valeur ajoutée initialement nul, un allègement de charges de 15 milliards d'euros financé ex ante, c'est-à-dire tel que

$$
\left(\left(1+\tau_{n q}\right) w_{n q} l_{n q}+\beta\left(1+\tau_{q}\right) w_{q} l_{q}\right) \hat{\tau}_{n q}=-p y \hat{t}=-15,
$$

conduit à une hausse de l'emploi de 420000 individus environ pour $\beta=0$ et de 60000 pour $\beta=1$. En termes d'emplois, la politique est donc un peu plus efficace que la TVA sociale (de 10 à 20000 individus), mais la hausse de l'emploi se fait cette fois-ci au détriment du capital : l'effet de substitution de travail nonqualifié au capital compense approximativement le moindre effet de demande lié à la baisse de l'investissement.

\section{Conclusion}

Ce texte a cherché à délimiter certaines conditions d'efficacité d'une politique de TVA sociale. Il insiste sur le rôle que semblent jouer les assiettes des différents prélèvements : la substitution d'une taxe sur la consommation à une taxe sur la masse salariale aurait un effet favorable sur l'emploi dès lors que la 
masse salariale est plus étroite que la consommation. Une maquette analytique calibrée suggère que l'emploi pourrait augmenter de 400000 à 500000 individus si les allègements étaient concentrés sur la main-d'oeuvre peu qualifiée, et que cette hausse serait très nettement amoindrie au fur et à mesure que la plage de salaires en bénéficiant est étendue.

Ce texte n'aborde pas, toutefois, la question de l'équité de ce type de mesure. Or, il est évident qu'un tel problème se pose, puisque la hausse des taux de TVA touche des individus qui ne sont pas concernés directement par les allègements. Aussi, une analyse de l'incidence fiscale de la politique serait utile et viendrait sans doute nuancer la seule considération de l'efficacité de cette réforme.

\section{Références}

[1] Audric, S., Givord, P. et C. Prost, Estimation de l'impact sur l'emploi nonqualifié des mesures de baisse des charges, Revue Economique 51, 2000.

[2] Chauvin, V. et alii, Evaluation du plan de relance de l'économie française présentée le 4 mai 2004 par M. N. Sarkozy, Document de travail OFCE 4, 2004.

[3] De Vreyer, P., Exonérations ciblées de cotisations sociales, Document de travail EPEE, Université d'Evry, 2002.

[4] Hervé, K., Estimation des élasticités du commerce extérieur sur l'ensemble des biens et services pour un panel de 17 pays, Economie et Prévision 147, 2001.

[5] Laffargue, J.P., Effets et financement d'une réduction des charges sur les bas salaires, Revue Economique 51, 2000.

[6] Lhorty, Y., Quand les hausses du SMIC réduisent le coût du travail, Revue Economique 51, 2000.

[7] Malinvaud, E., Les cotisations sociales à la charge des employeurs : analyse économique, Rapport du CAE 9, 1998.

[8] McLure, C., General equilibrium incidence analysis, Journal of Public Economics 4, 1975.

[9] Skalitz, A., Les salaires dans l'industrie, le commerce et les services en 2002, INSEE Résultats 38, Décembre 2004.

[10] Salanié, B., Une maquette analytique de long terme du marché du travail, Economie et Prévision 146, 2000. 


\section{Annexe 1. Le coût d'usage du capital}

A la date $s$, en choisissant le bien comme numéraire, c'est-à-dire en posant le prix-consommateur égal à 1 , la recette est égale à $f\left(k_{s}, l_{s}\right) /(1+t)$. Le coût du travail est égal à $(1+\tau) w_{s} l_{s}$ et le coût d'achat des biens d'équipement s'élève à $i_{s} /(1+t)$, puisque ces biens sont payés au prix-producteur (hors-taxe). Pour un taux de dépréciation $\delta$, on a $k_{s+1}=k_{s}+i_{s}-\delta k_{s}$. En notant $r$ le taux d'intérêt réel, le problème de l'entreprise s'écrit donc :

$$
\max _{\left(k_{s}, l_{s}\right)_{s \geq 0}} \sum_{s=0}(1+r)^{-s}\left(f\left(k_{s}, l_{s}\right)-(1+t)(1+\tau) w_{s} l_{s}-\left(k_{s+1}-(1-\delta) k_{s}\right)\right),
$$

où $k_{0}$ est donné. La condition du premier ordre par rapport à $l_{s}$ s'écrit :

$$
f_{l}\left(k_{s}, l_{s}\right)=(1+t)(1+\tau) w_{s} .
$$

La condition du premier ordre par rapport à $k_{s}$ s'écrit :

$$
\begin{gathered}
-(1+r)^{1-s}+(1+r)^{-s}\left(f_{k}\left(k_{s}, l_{s}\right)+(1-\delta)\right)=0 \\
\Leftrightarrow f_{k}\left(k_{s}, l_{s}\right)=r+\delta .
\end{gathered}
$$

Dans le cas d'un basculement sur la valeur ajoutée, le problème de l'entreprise s'écrirait :

$\max _{\left(k_{s}, l_{s}\right)_{s \geq 0}} \sum_{s=0}(1+r)^{-s}\left(f\left(k_{s}, l_{s}\right)-(1+t)(1+\tau) w_{s} l_{s}-(1+t)\left(k_{s+1}-(1-\delta) k_{s}\right)\right)$,

de sorte que le coût d'usage du capital serait égal à $(1+t)(r+\delta)$, et non pas simplement à $r+\delta$. 